Supporting Information for:

\title{
Spatiotemporal Tracking of Brain Tumor-Associated Myeloid Cells In Vivo through Optical Coherence Tomography with Plasmonic Labeling and Speckle Modulation
}

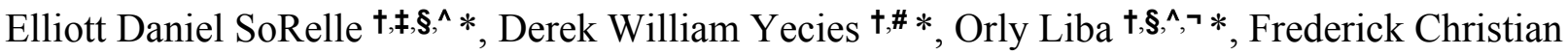 \\ Bennett ${ }^{\dagger}$, Claus Moritz Graef ${ }^{\#}$, Rebecca Dutta ${ }^{\dagger, \S^{\wedge},}$, Siddhartha Mitra ${ }^{\#, \diamond, 1}$, Lydia-Marie Joubert ${ }^{\Delta, 2}$, \\ Samuel Cheshier $\#, \diamond, 3$, Gerald A. Grant $\#, \diamond, \&$ Adam de la Zerda $†, \neq, \S^{\wedge}, \neg, \sim *$ \\ †Department of Structural Biology, ‡Biophysics Program, §Molecular Imaging Program (MIPS), \\ ${ }^{\wedge}$ Bio-X Program, \#Department of Neurosurgery, Division of Pediatric Neurosurgery, \\ ᄀDepartment of Electrical Engineering, ${ }^{\diamond}$ Institute for Stem Cell Biology and Regenerative \\ Medicine and the Ludwig Cancer Center, ${ }^{\Delta}$ Cell Sciences Imaging Facility, \\ Stanford University, 299 Campus Dr., Stanford, CA 94305, USA \\ The Chan Zuckerberg Biohub, 499 Illinois St., San Francisco, CA 94158, USA \\ * Equal contribution \\ *To whom correspondence should be addressed: adlz@stanford.edu
}

\footnotetext{
${ }^{1}$ Present address: University of Colorado Anschutz Medical Campus, Department of Pediatrics, Mail Stop 13001 E. 17 $7^{\text {th }}$ P1, B119, Aurora, CO 80045, USA

${ }^{2}$ Present address: Stellenbosch University, Chamber of Mines Building, 7600 Stellenbosch, South Africa

${ }^{3}$ Present address: University of Utah Primary Children's Hospital, Department of Pediatric Neurosurgery, 100 N Mario Capecchi Dr., Suite 3850, Salt Lake City, UT 84113, USA
} 


\section{Supporting Note on Terminology}

OCT contrast - this term refers to the (log-scale) intensity in traditional OCT images, which is typically depicted in grayscale. OCT contrast originates from light scattering in tissue.

Scattering intensity - scattering intensity is similar to OCT contrast, but typically is used in the context of describing the signal originating from the optical properties (ex: scattering crosssection) of an imaging contrast agent (in this manuscript, LGNRs)

Spectral contrast/signal - the spectral contrast of a species in an OCT image describes the result of processing raw spectral domain OCT intensity images using a dual-band method to produce a difference image. This can be used to distinguish species that scatter different wavelengths within the source bandwidth (for example, to distinguish LGNRs from tissue). Spectral scattering - the unique scattering spectrum (or spectral scattering) form LGNRs is what enables their distinction from the tissue scattering spectrum through the use of spectral contrast methods, as described above.

LGNR contrast/signal - this term is closely related to the spectral scattering of LGNRs, although it generally describes both the spectral signature scattering intensity produced by LGNRs (as described in the main text, the intensity multiplied by the difference signal that is calculated from the dual-band spectral processing method.

\section{Supporting Discussion}

\section{Animal-to-animal variability in LGNR detection}

Generally, some degree of variability in LGNR uptake and retention across animals should be expected. Such variability may arise from differences in the degree of tumor vascularization and the extent of extravasation of the particles from circulation. Extravasation is partially mediated by vascular pore size and the extent of tumor infiltration and disruption of the surrounding vasculature. We expect each of these factors to vary across animal replicates because of 1) the context-dependent and idiosyncratic growth of each tumor and 2) the inherent variability in tumor location, residual hydrostatic pressure, and other physical factors resulting from the tumor model preparation methods. To better dissect the sources of variation, we further report quantification from each mouse at each time point, with and without normalization for tumor volume (Figure S4). We note that accounting for tumor volume reduces, but does not eliminate, the extent of variability with respect to detection of vasculature and retention of LGNRs. Interestingly, while differences in the extent of vascularity is 
evident from post-injection images, these differences are not necessarily correlated with the extent of LGNR uptake observed at $40 \mathrm{~h}$. For example, Mouse 3 exhibits the highest vascularity density (i.e., after volume normalization), yet also has the lowest measured LGNR retention at $40 \mathrm{~h}$. This would suggests a greater degree of tumor vascularization, but less vessel permeability, leading to reduced EPR.

We also observed some variation in the amount of background signal prior to LGNR injection across images from mice processed with the optimized thresholding and segmentation parameters. For example, the pre-injection image from Mouse 2 exhibits more background signal (false positives) than either of the other two mice, despite the absence of LGNRs at this time point (Figures S1-S4). While this higher initial background can be accounted for in quantification, it is worth considering the origin of the phenomenon. As described in the main text, every OCT image has some amount of speckle noise. For SD-OCT images, some portion of that speckle noise will, by definition, be spectral speckle noise. Further, it is conceivable that, consistent with Mie Theory, tissue microstructures of certain sizes may have more or less spectral bias with respect to light scattering due to differential interaction with various wavelengths of light. Thus it may be expected that tumors (or other tissues) will exhibit variations in background spectral signals due at least in part to the complex nature of the interactions of light with distinct tissue features at the relevant scale. In a non-technical sense, these differences may be understood as somewhat analogous to the well-characterized birefringent properties of different cell layers and microstructures within the same tissue observable with OCT.

\section{LGNR circulation properties and timescale of accumulation in glioblastoma}

We have previously observed that intravenously-administered LGNRs produce uniform, maximized signal in circulation within several minutes after injection, and that the circulation half-life of these particles is approximately $18 \mathrm{~h}^{21}$. This extended circulation affects the ability to assess the timeline of extravasation and uptake in this particular experimental design. LGNRs within the 
vasculature of each tumor can be detected within minutes; however the persistence of LGNR signal in circulation is expected to impede the ability to accurately quantify extravasation over time.

Distinguishing LGNRs still in circulation from those that have extravasated poses a challenge to accurate quantification from an image processing standpoint. Moreover, the ability to detect LGNRs is mediated by both the rate of extravasation and the rate of phagocytic uptake by macrophages, as each of these factors determine the extent to which LGNR signal rises above the limit of detection, which we have previously defined for LGNRs in various biological media and tissues ${ }^{19,42}$. However, the gradual nature of LGNR accumulation necessarily means that the early phases of extravasation would be extremely challenging to characterize, since levels of LGNRs within the interstitium and/or individual TAMs would be too low to reliably detect. Thus, an attempt to characterize the timescale of LGNR uptake may be expected to exhibit artificial time delays.

\section{Factors affecting nanoparticle uptake in tumors}

We chose to use LGNRs in this study because they provide optimized and distinct spectrallyresolvable contrast in SD-OCT imaging. Generally, LGNRs provide more contrast than conventional GNRs due to increased scattering, more contrast than gold nanospheres due to the location of the plasmonic resonance with respect to the illumination bandwidth, and more unique spectral contrast with other near-infrared plasmonic shapes such as nanoshells or nanostars, which have broad spectral bands rather than narrow peaks (broad scattering over the entire illumination bandwidth leads to poor spectrality due to low difference signals after dual-band signal processing). However, particles of different architectures may also be explored in the future. Previous studies have shown that both particle size $\mathrm{S}^{\mathrm{S}}$ and tumor type $\mathrm{S}^{\mathrm{S}}$ can affect the extent of nanoparticle extravasation and retention. It should be noted that, based on the relative signal intensities of different particles, it is conceivable that a particle with greater accumulation but poorer image contrast may be undetectable, whereas a particle with less accumulation and greater image contrast may be more readily detected. 


\section{SEM imaging considerations}

With respect to SEM characterization, the most reliable indicator of LGNR uptake in discrete cells is the high contrast from gold, not abundant observation of the uniform rod shapes (although individual particles can be observed in some instances, as in Figure S6d). This is for several reasons: 1) Whereas the osmium tetroxide, uranyl acetate, and lead citrate stains provide low to medium contrast across entire tissue sections, only the presence of gold in high concentrations can account for the highintensity staining observed in a small subset of cells within tumor sections. 2) The nature of the sample and its preparation contribute to the lack of frequency with which individual rods are observed. Rods in the tissue section have three degrees of freedom with respect to orientation - thus, there should be relatively few LGNRs oriented perfectly planar to the sample surface, which is required to observe the shape. Furthermore, the sample preparation (especially the mechanical forces from microtome sectioning) can be expected to change or disrupt the shapes of LGNRs embedded within tissue at or near the sectioning plane. Note that SEM was chosen instead of TEM in part due to the additional modes of analysis used for tissue samples resected from the same animals (e.g., HSM-AD, immunofluorescence), which require sample mounting onto microscope slides.

\section{Supporting References}

S1. Tong, X.; Wang, Z.; Sun, X.; Song, J.; Jacobson, O.; Niu, G.; Kiesewetter, D.O.; Chen, X. Size Dependent Kinetics of Gold Nanorods in EPR Mediated Tumor Delivery. Theranostics 2016, 6, 2039-2051. 
S2. Smith, B.R.; Kempen, P.; Bouley, D.; Xu, A.; Liu, Z.; Melosh, N.; Dai, H.; Sinclair, R.; Gambhir, S.S. Shape Matters: Intravital Microscopy Reveals Surprising Geometrical Dependence for Nanoparticles in Tumor Models of Extravasation. Nano. Lett. 2011, 12, 3369-3377. 


\section{Supporting Figures}

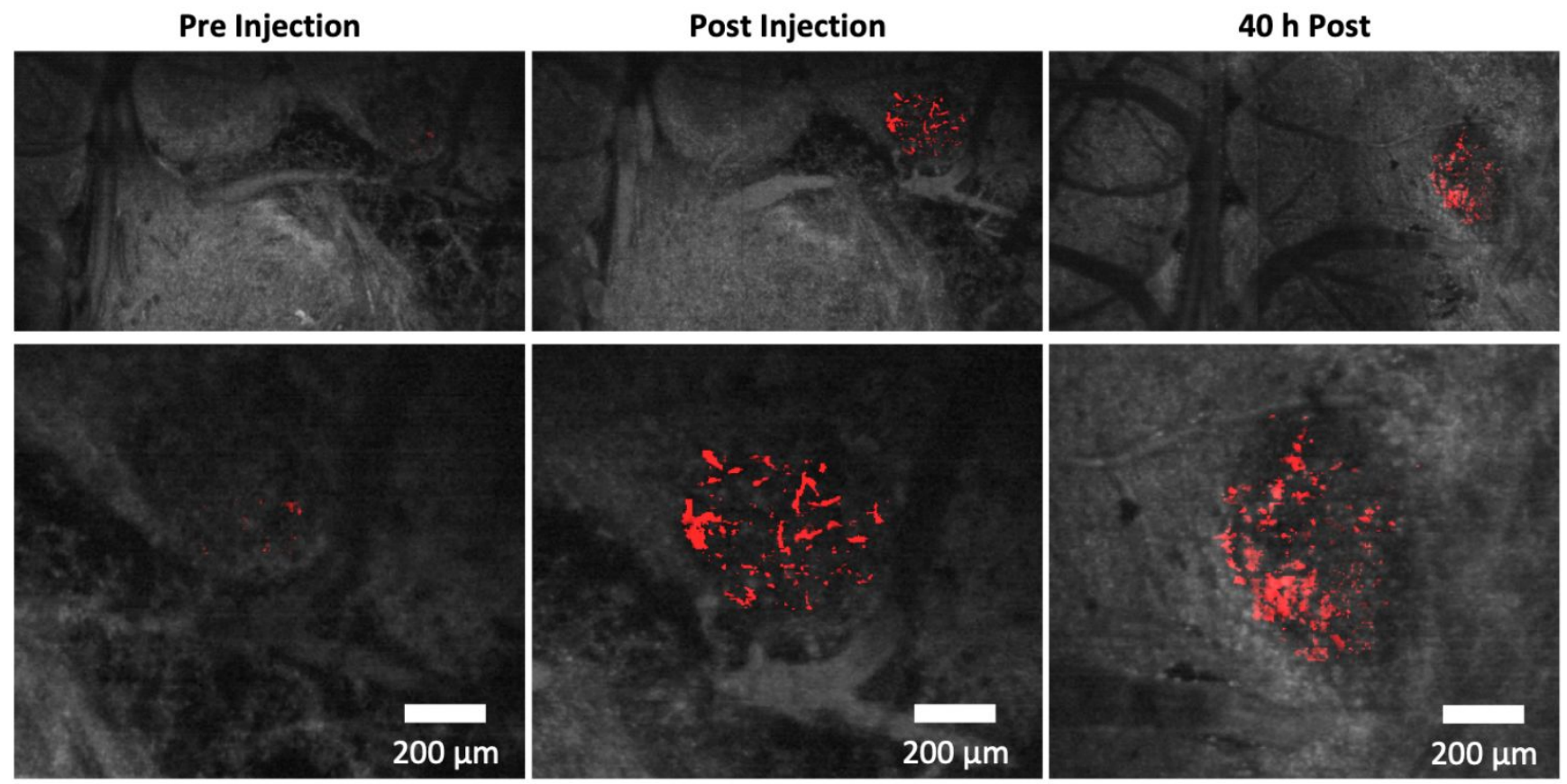

Figure S1. Detail of segmented detection images from Mouse 1.
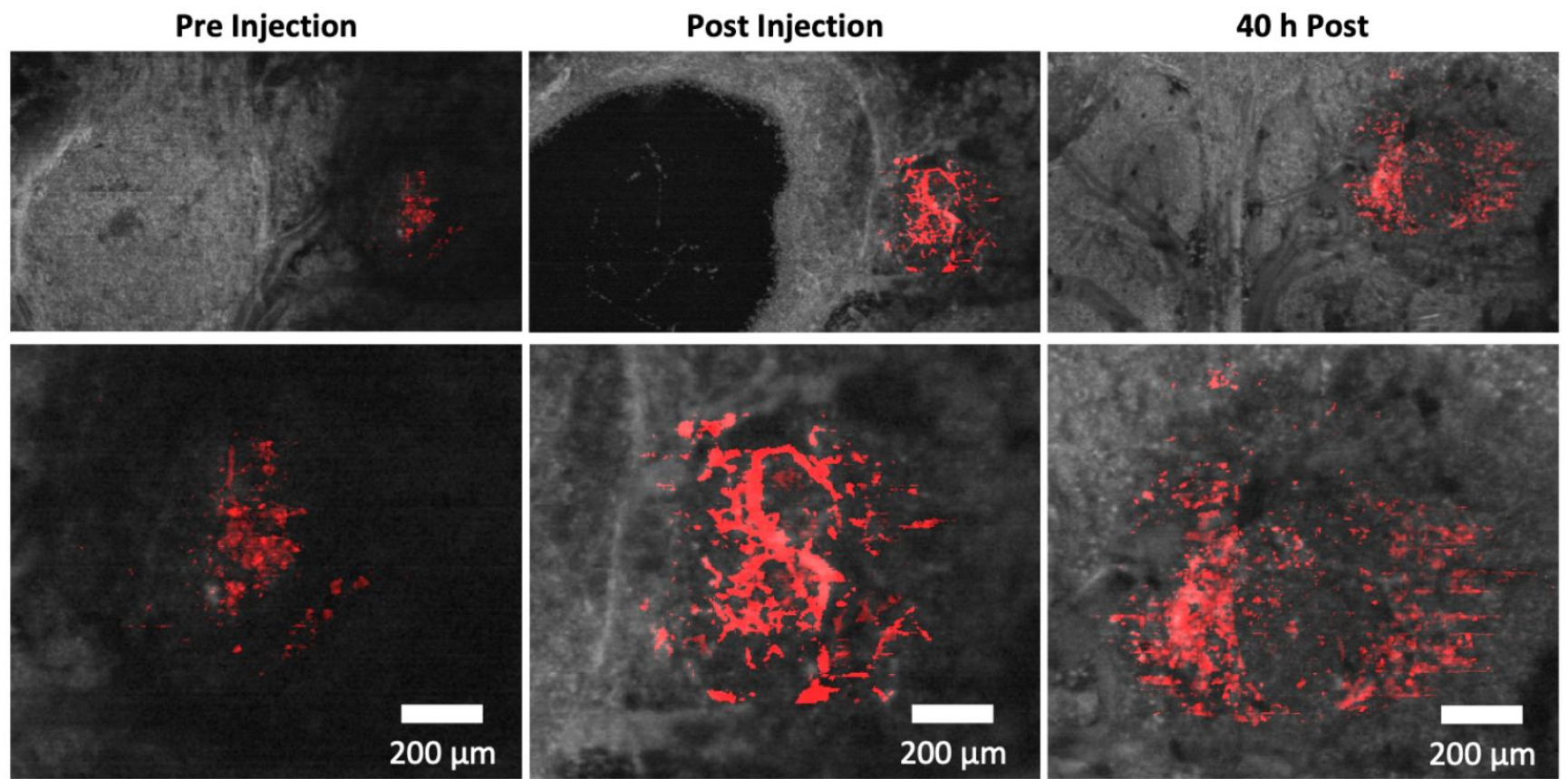

Figure S2. Detail of segmented detection images from Mouse 2. 

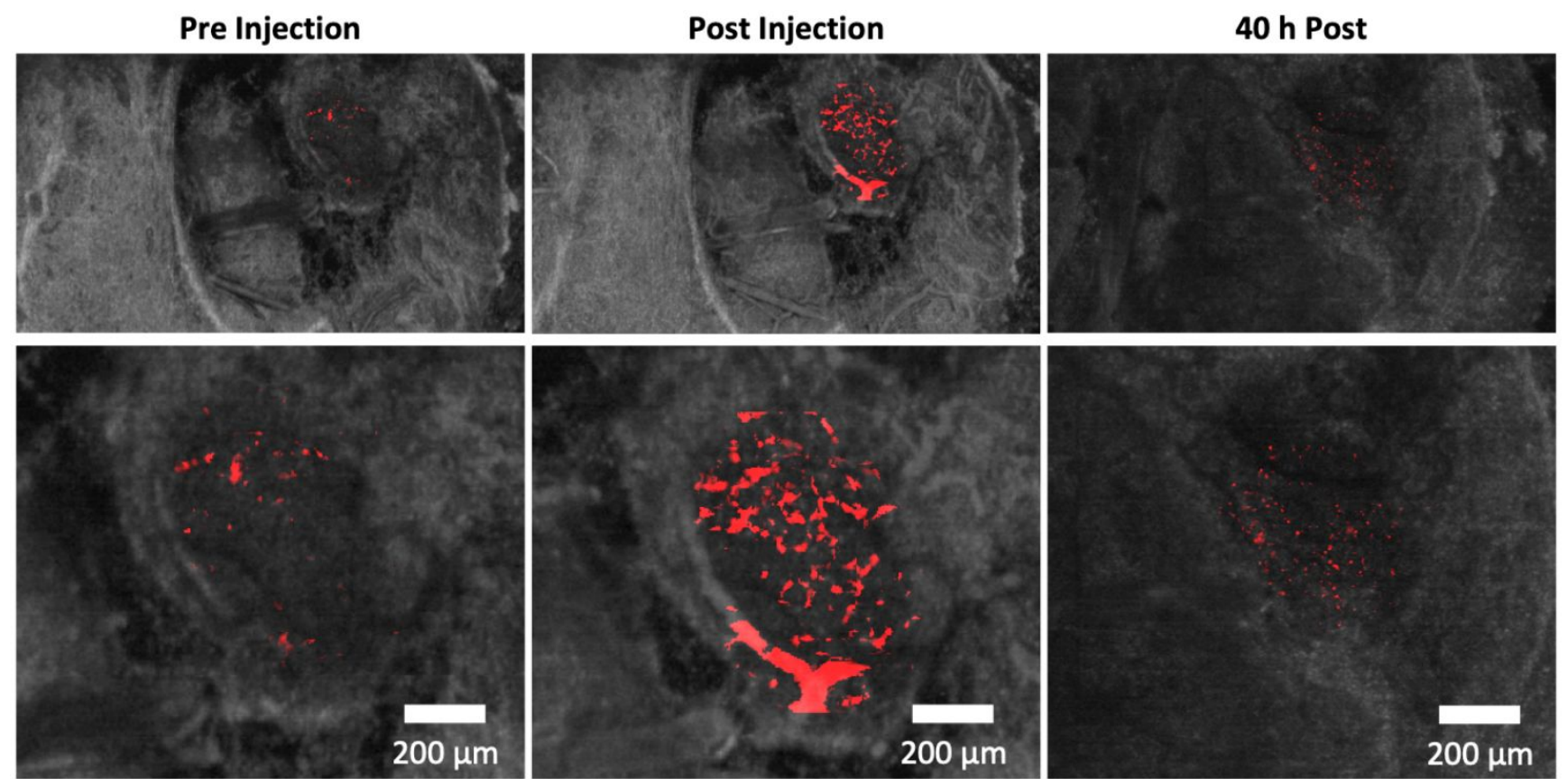

Figure S3. Detail of segmented detection images from Mouse 3.

a

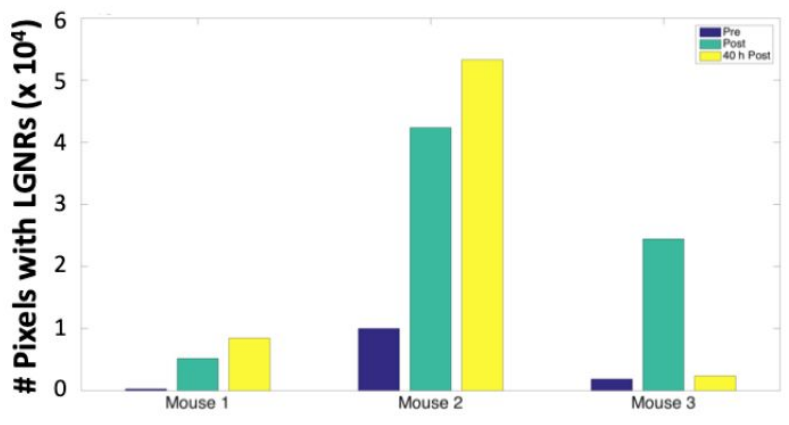

b

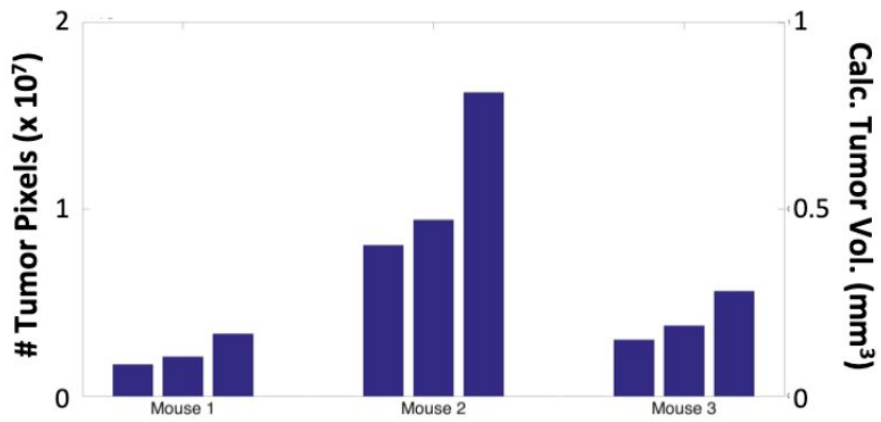

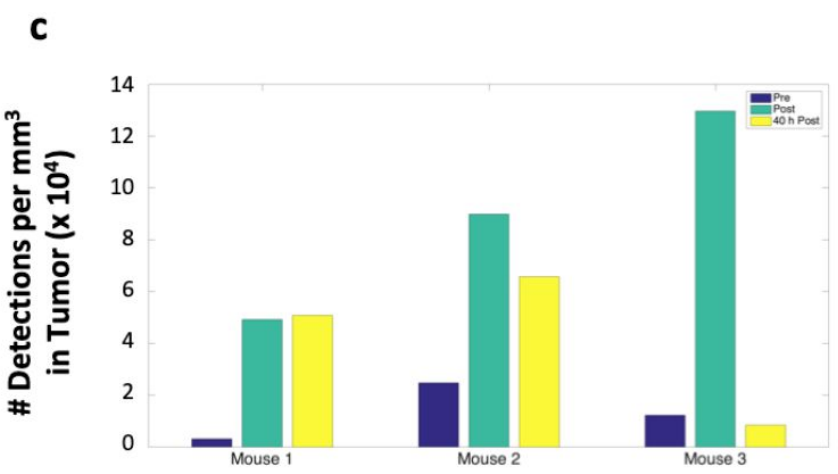

Figure S4. Quantification of LGNR detection for individual mice. (a) The raw number of detected pixels for each mouse at each time point. (b) The size of each tumor at each time point, measured as a number of pixels via tumor delineation from healthy tissue and converted to calculate tumor volume by the known pixel (voxel) size. (c) Volume-normalized number of detections for each mouse and time point. 
a

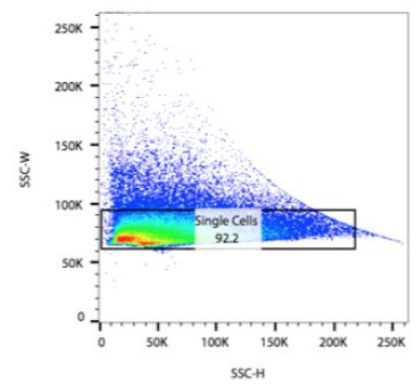

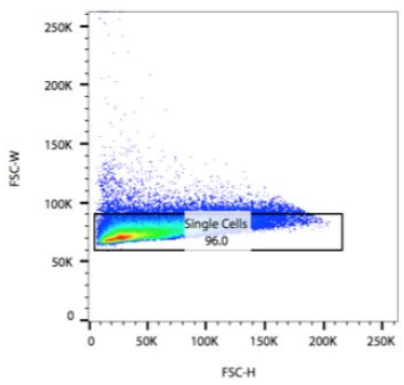

b
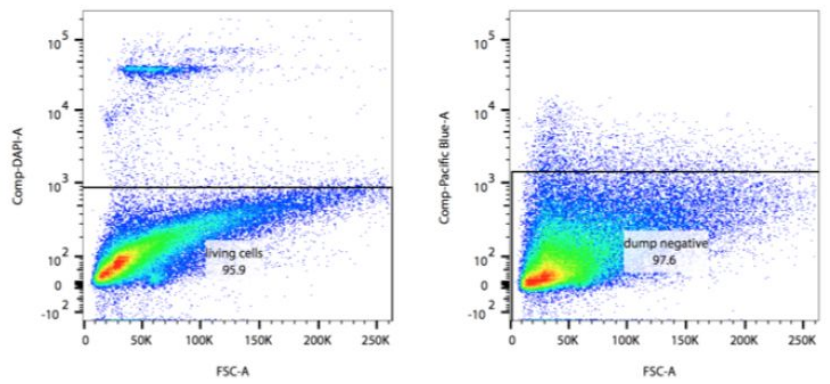

c
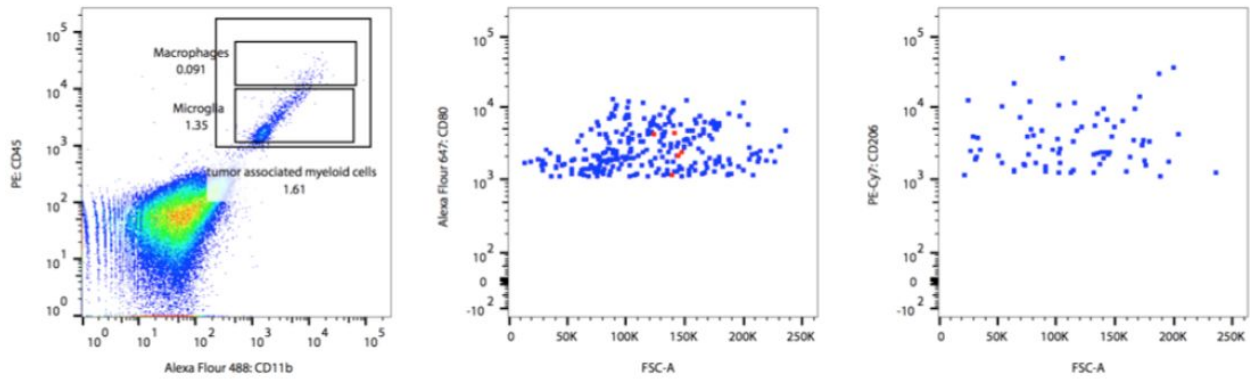

Figure S5. Additional details of gating used for FACS. (a) Gating used for single cells. (b) Gating used for live/dead cells. (c) Gating used for resolving macrophages and microglia. 
a

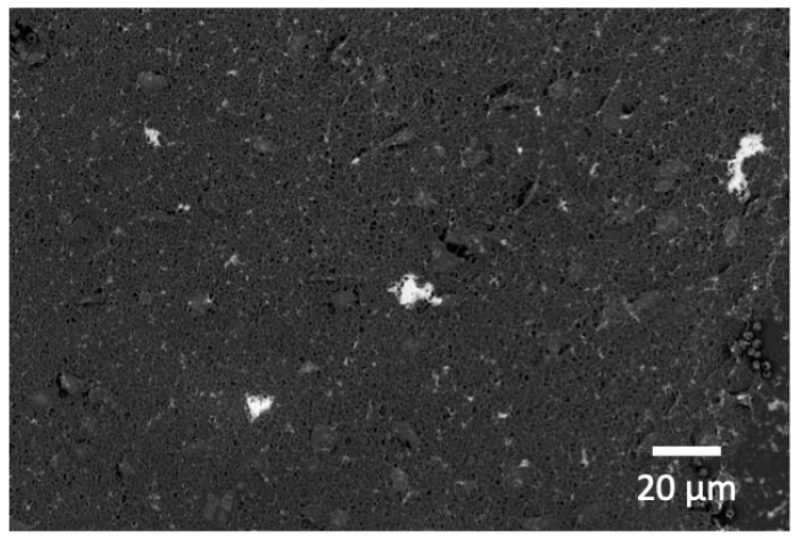

c

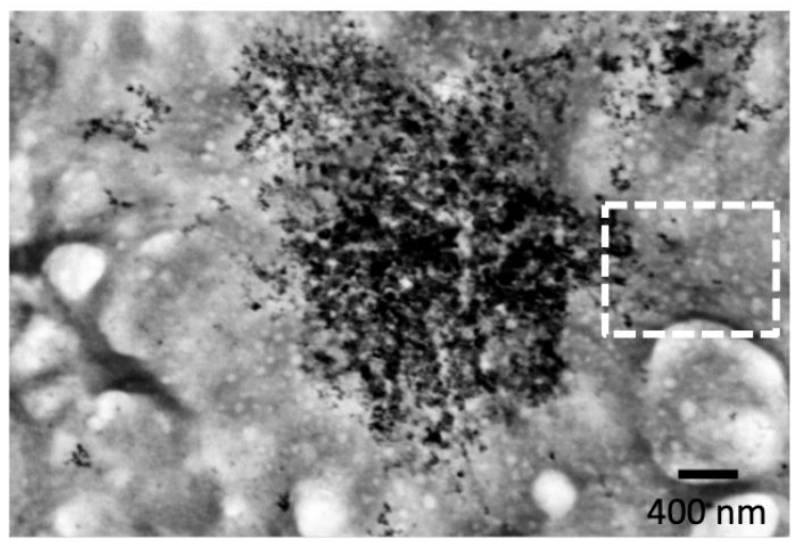

b

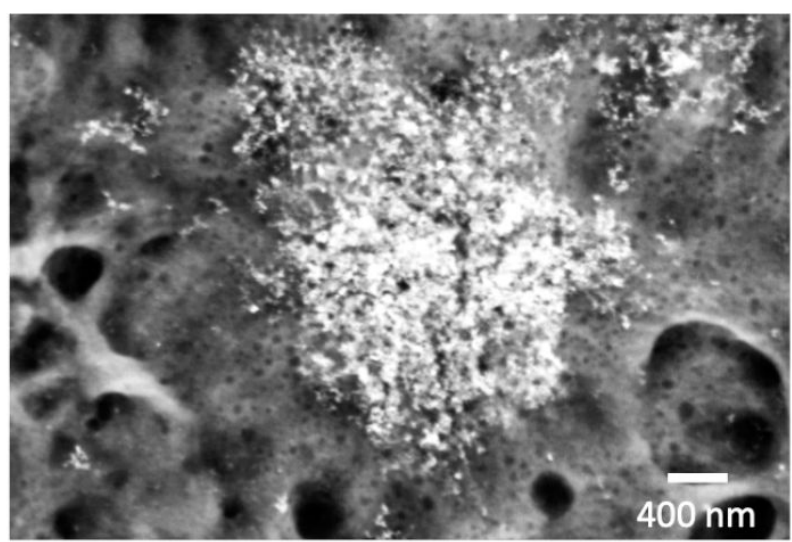

d

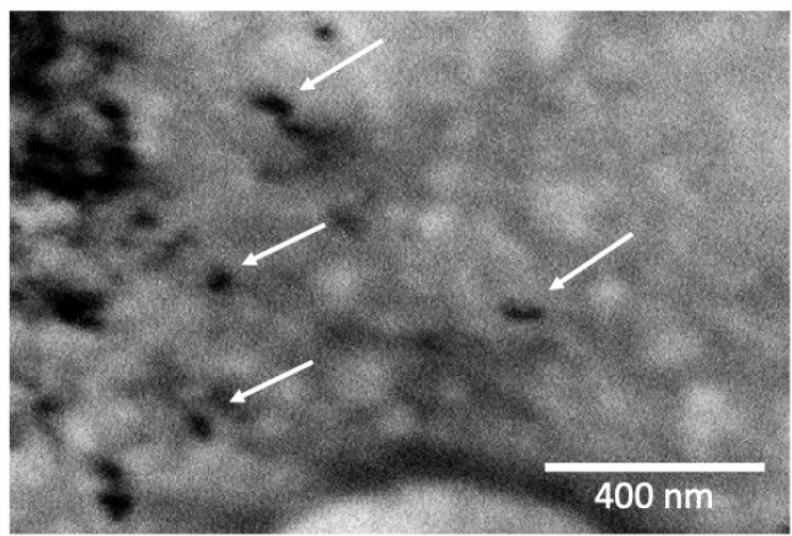

Figure S6. Additional SEM characterization of LGNR uptake in TAMs and activated microglia. (a) Individual cells loaded with LGNRs can be observed via the high contrast of concentrated gold against the tissue background. (b) Higher magnification image of a single macrophage/microglia loaded with LGNRs. (c) As in (b), but presented with inverted contrast. (d) Zoom detail of dashed area in (c) showing putative individual LGNRs. The processing of the tissue sections, especially the mechanical force of cutting sections with a microtome, are expected to alter the morphology and appearance of LGNRs to some degree. Thus, intact LGNRs embedded parallel to the section surface are expected to be infrequent throughout the macrophages/microglia. 
a
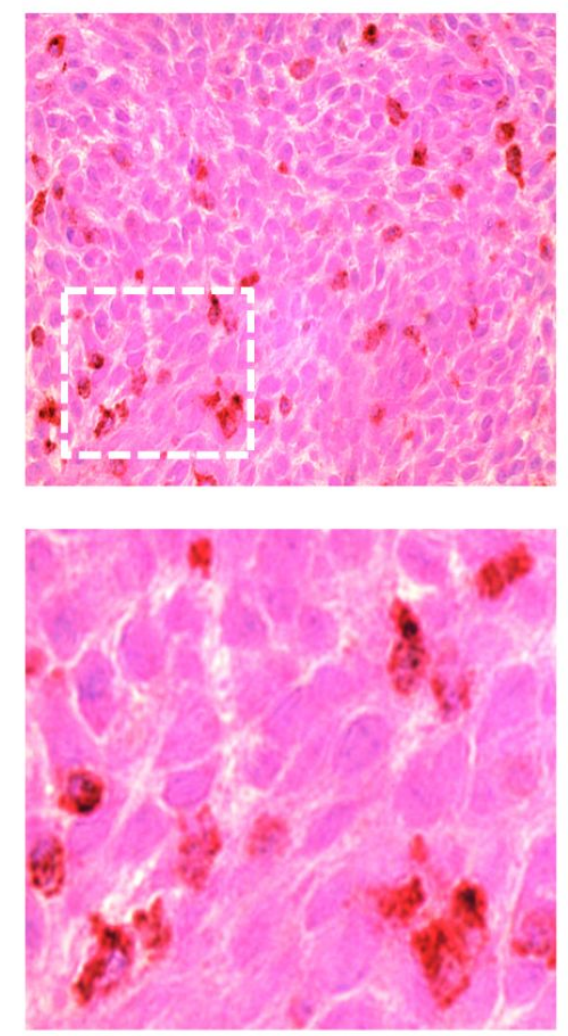

b
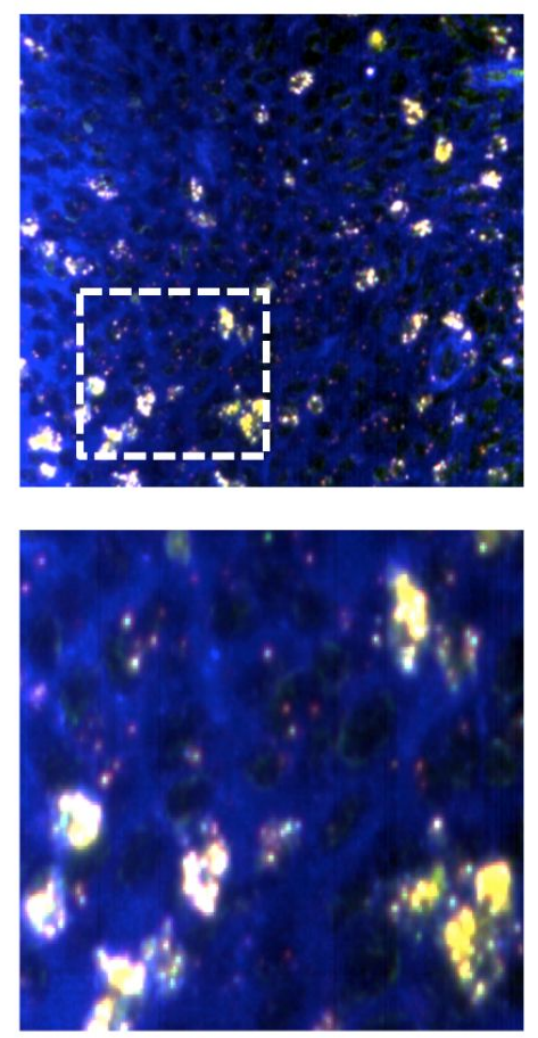

C
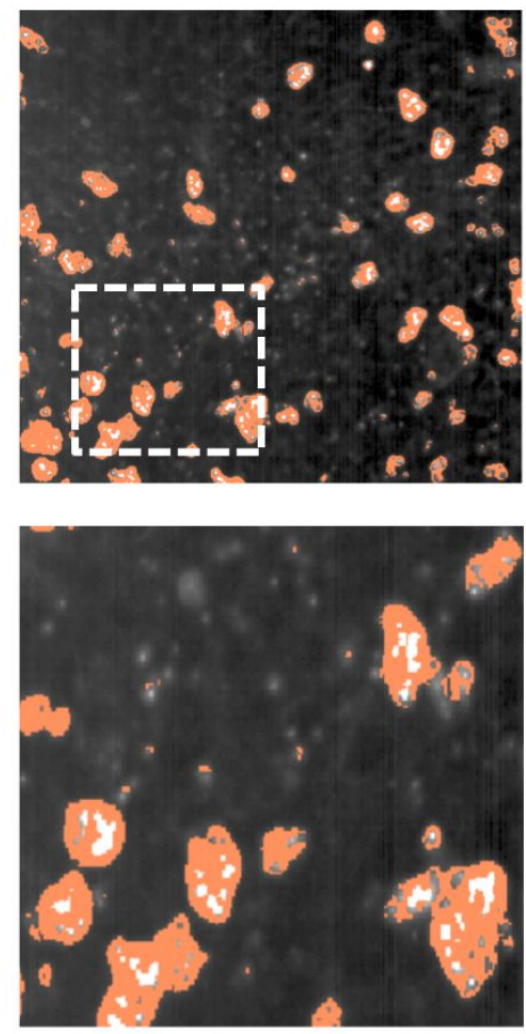

Figure S7. Additional detail images for co-registration of CD68 staining and LGNR uptake in tumor tissue. (a) Contrast and brightness-adjusted image of tumor tissue with H\&E, CD68 HRP stains and zoom. (b) Contrast and brightness-adjusted image of the same region imaged via hyperspectral darkfield microscopy. (c) Detection image of LGNRs by plasmonic scattering signature in the hyperspectral image ( $\mathrm{LGNR}^{+}$regions denoted in orange). 
a
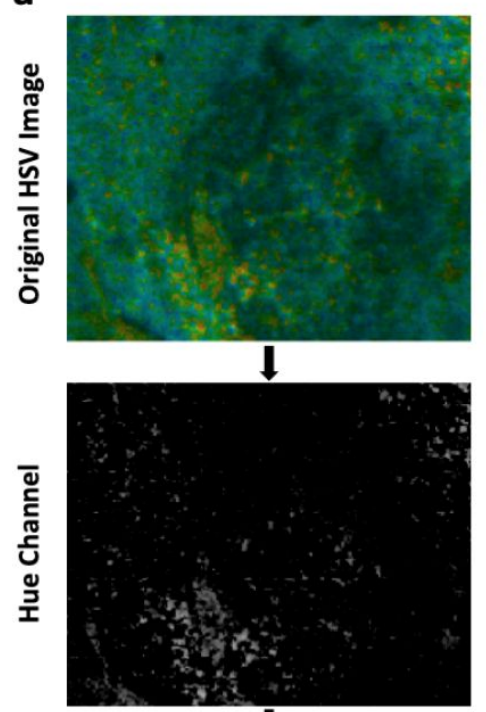

I

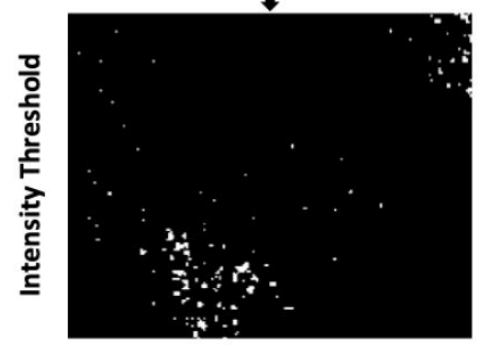

b
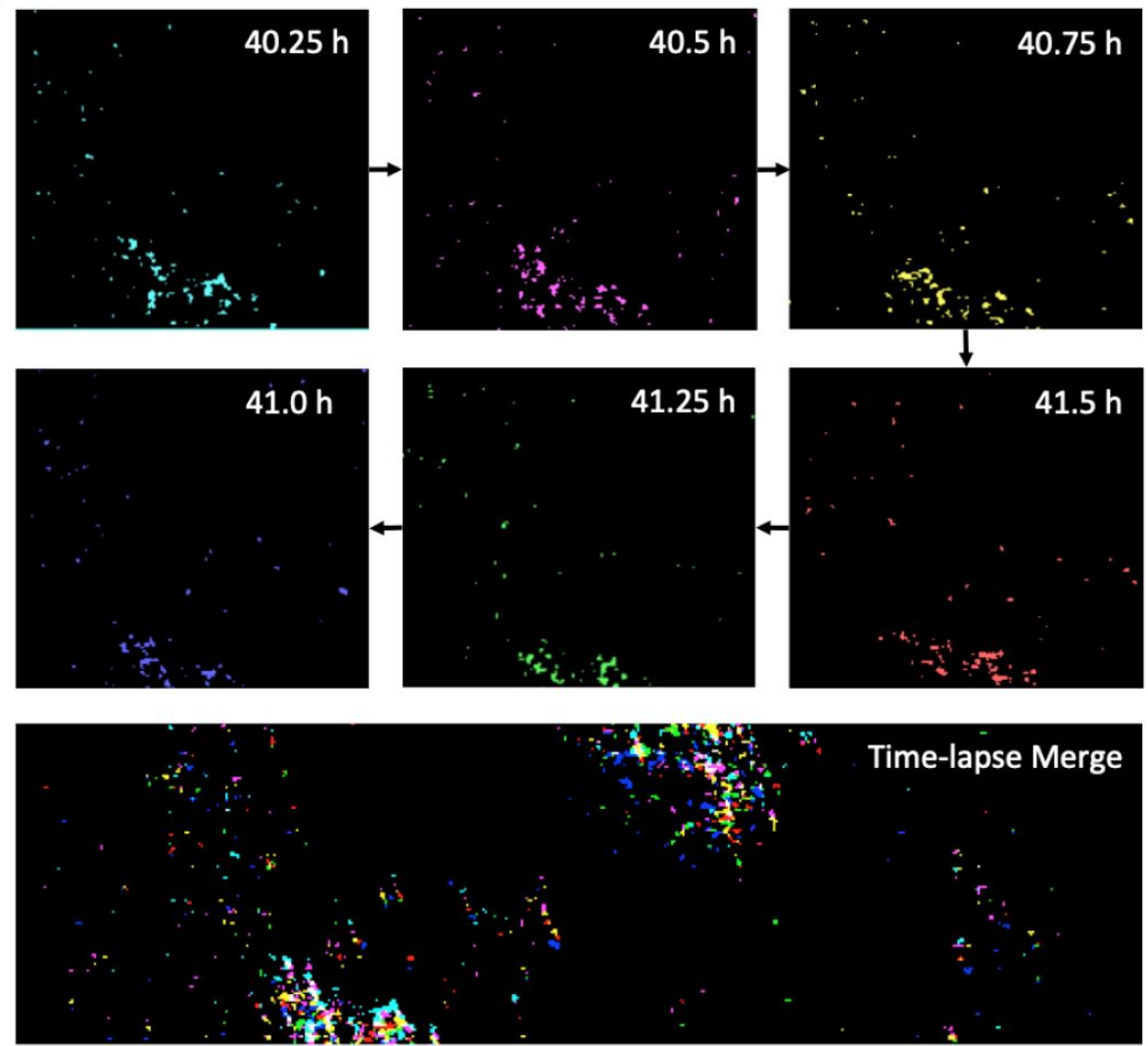

Figure S8. Alternate visualization of LGNR-loaded cell migration over time. (a) Original HSV format spectral contrast images can be processed to yield clearer visualization of $\mathrm{LGNR}^{+}$pixels by isolating and thresholding the hue channel (LGNRs exhibit a distinct hue relative to surrounding tissue in the parula color map renderings). (b) Threshold images of the hue channel from the mouse shown in Figure 5a,b. Each time point is false-colored, then overlaid as a time-lapse merge to demonstrate clear migration of individual loaded cells over time. 